\title{
La solitude de l'homme moderne chez Le Clézio: étude appliquée à Désert et à Mondo et autres histoires
}

Galal Abdelnaser Aboudeif Ali-

Assistant au département du Français.

\section{Introduction}

Une des menaces qui mettent en péril la vie de l'Homme au sein de la société moderne, la solitude est une impression fortement vécue par la plupart des hommes dans les milieux urbains. Rainer Maria Rilke décrit la solitude de l'homme moderne en disant :

«L'homme n'est plus l'être de société qui marche en équilibre parmi ses semblables, [...] il n'est plus non plus celui à qui s'adresse l'alternance du soir et du matin, du proche et du lointain. Il est placé parmi les choses comme une chose, infiniment seul [...] tout ce qu'ils avaient de commun s'est retiré des choses et des hommes pour se réfugier dans la commune profondeur où s'abreuvent les racines de tout ce qui est en croissance» ${ }^{1}$

Dans cette présente étude nous essayerons de repérer la problématique de la solitude de l'homme moderne au sein d'une société hyper modernisée et qui se caractérise par l'abus de la technologie, tout en se basant sur les deux œuvres majeures de J.M.G., Le Clézio; à savoir: Désert et Mondo et autres histoires.

Mais pourquoi Le Clézio ? En effet, Le Clézio se considère comme : «Un écrivain de la rupture, de l'aventure poétique et de l'extase sensuelle, l'explorateur d'une humanité au-delà et en dessous de la civilisation régnante ${ }^{1}$, c'est en ces termes que le Jury du Prix Nobel qualifiait J. M. G. Le Clézio en 2008 pour lui accorder cette prestigieuse récompense. Le Clézio qualifie lui-même comme «un écrivain qui a du mal à vivre dans le monde moderne $»^{2}$

Du point de vue de notre romancier Le Clézio, la culture moderne, en changeant les habitudes de l'Homme qui vivait en paix au sein de la nature, pousse l'Homme à se sentir isolé dans une société de consommation. Il ne devient pas réellement capable à communiquer avec ses semblables.

"L'isolement est un fait, une donnée objective qui peut s'accompagner d'un ressenti, la solitude qui résulte de la souffrance liée à l'impossibilité d'échanger, de discuter ou de partager ses émotions et son vécu». ${ }^{3}$

En plus, Le Clézio estime que l'Homme ne peut transmettre ses intentions ou ses messages par la communication, celle-ci étant tenue pour la cause fondamentale du malentendu aux yeux de Le Clézio. Ainsi, chaque personnage se trouve totalement exclu, d'une exclusion, voire d'une solitude, qui se voit fortifié par l'incommunicabilité et par l'impossibilité du dialogue. On a l'impression qu' «on vit dans un désert [...] et on est là, dans sa bulle, à crier pour les sourds». ${ }^{4}$

La solitude est un thème récurrent dans l'œuvre le clézienne. Certains critiques concernés à l'étude de l'œuvre le clézienne ont mis en lumière «les principaux 
éléments fondateurs des récits le cléziens : le désert, le silence, la nature, sans toutefois les relier à la source qui les ressemble tous : la solitude». ${ }^{5}$

Comme le constate Ruth Amar : "L'œuvre le clézienne traduit la nouvelle société humaine à la fin du XXème siècle, composée d'hommes à l'écart, écrasés par l'emprise de la technologie. Cet écart est parmi tant d'autres qui appartiennent tous à la même société. Le drame de la condition humaine tel qu'il est exhibé dans l'œuvre, tient au fait que l'homme occidental est obsédé par son désir de suprématie sur la nature et la matière, ressentant un besoin continuel de disséquer le monde afin de pouvoir expliquer. Ce faisant, l'homme s'est mis en solitude, coupant ses liens avec le monde naturel» ${ }^{6}$

D'ici, on peut dire que la solitude représente l'axe de la plupart des œuvres de Le Clézio. C'est un syndrome qui hante les personnages de ses récits. En outre, tous ces éléments fondateurs ne sont que les propres composants de la solitude.

Avant de mettre en exergue les structures de la solitude dans deux œuvres majeurs de Le Clézio, Désert et Mondo et autres histoires, nous allons mettre en lumière les différentes définitions de la solitude en tant que raison, voire une motivation, du refus de la modernité et du retour à la nature dans l'œuvre le clézienne.

\section{Qu'est-ce que la solitude?}

Il est à signaler d'abord que, dans la plupart des langues, le champ sémantique de la solitude comporte deux termes étroitement liés : solitude et isolement. Le mot solitude est emprunté d'un mot latin «solitudo» qui est le synonyme d'un lieu désert, vie isolée, état d'abandon, et de «solus» qui signifie absence, manque. Le mot isolement est un dérivé du verbe «isoler», lui-même issu de l'adjectif isolé, lequel vient de l'italien qui signifie isolato signifiant construit en ilôt, séparé.

Selon des psychiatres, la solitude est un comportement auquel recourt l'homme pour échapper à l'emprise de la vie. «En psychanalyse l'isolation est un mécanisme de défense qui consiste à isoler une pensée ou un comportement, pour éviter toutes les connexions à d'autres pensées ou comportements. Il s'agit d'une rupture de la chaîne associative d'une pensée ou d'une action»?

La solitude peut être définie, selon le constat de Marie-Chantal Doucet ${ }^{8}$, par «la perte du sens». Aussi, nous citerons de May Rollo, qui en analysant la situation de l'homme moderne, exprimait la solitude de celui-ci en la reliant à la perte de ses relations avec la nature.

"[...] la solitude [...] est l'ultime conséquence de quatre siècles de travail de la séparation de l'homme en tant que sujet du monde objectif. Cette solitude s'est exprimée pendant plusieurs siècles par la passion de l'homme occidental d'exercer son pouvoir sur la nature, mais se montre maintenant dans un détachement total de la nature et dans un sentiment, vague, inarticulé, de désespoir d'atteindre de vraies relations avec le monde de la nature» ${ }^{10}$

\section{Le personnage le clézien, un personnage solitaire}

La critique journalistique a longtemps présenté Jean-Marie Gustave Le Clézio comme «un écrivain secret, un homme de silence toujours soucieux de se tenir à l'écart des modes et des mondanités littéraires, et cultivant par une sorte d'atavisme le goût d'une solitude essentielle» ${ }^{11}$. N'avait-il pas justement fait l'éloge de la solitude à travers l'œuvre de Michaux à l'occasion de son mémoire de diplôme? C'est 
en ces termes que J.M.G. Le Clézio introduit son mémoire qui s'est intitulé Le thème de la solitude dans l'œuvre d'Henri Michaux pour exprimer le syndrome de la solitude de l'homme du vingtième siècle.

«Aujourd'hui, dans un monde livré à la multiplicité et à l'indéfini, à l'écroulement, à une sorte d'apocalypse immanente et comme déjà imparfaite, il semble qu'il n'y ait plus place, en fait de vérité, que pour une immense et fanatique solitude. C'est cet isolement, dont la prise de conscience s'est faite lentement, qui constitue le thème fondamental de notre époque. L'homme. Privé d'unité, déséquilibré, dépossédé de lui-même, se trouve tel qu'il était au commencement: en proie à deux terreurs, marqué par l'angoisse, pressentant des dangers et des gouffres qu'il ne peut comprendre. Il recherche des frères afin d'atténuer, en la partageant, la crainte qui l'habite. Mais il ne sait trouver que des ennemis» ${ }^{12}$.

Comme ses personnages qui sont son alter ego, Le Clézio est un écrivain solitaire. A partir de l'âge de sept ans il préférait rester à la maison pour écrire plutôt que d'être en compagnie d'autres enfants de son âge. "C'était une habitude que j'avais prise. Au lieu de m'amuser, au lieu de faire l'effort [...] d'être comme tout le monde, je préférais rester chez moi à écrire» ${ }^{13}$. C'est l'un des formes positives de la solitude. Solitude qui pousse à contempler, à créer, à écrire.

Certains critiques ont qualifié J.M.G Le Clézio comme le romancier de la solitude et de l'errance. En outre, le personnage le clézien peut être défini, d'une manière générique, comme un personnage solitaire évoluant en marge de la société. Ce ne sont pas des personnages exclus mais des marginaux volontaires. Au lieu d'affronter les défis et les maux de la société moderne, les personnages de Le Clézio choisissent de vivre avec eux-mêmes. Pour eux, vivre à l'écart est une volonté ardente, une solution pour échapper à cet enfer moderne.

«Le personnage central des récits le cléziens se construit en solitude, mais une solitude ambiguë. Elle est reliée à un processus de modernisation, de submergement dans la vie moderne qui sera surtout mis en lumière dans l'espace urbain» ${ }^{14}$

Lalla, le personnage central autour duquel se nouent et dénouent l'intrigue dans Désert, est une adolescente orpheline qui subit la solitude dès sa naissance. «Son père est mort dans un accident avant sa naissance, sa mère, Hawa, est morte dans les fièvres peu de temps après sa naissance». ${ }^{15}$ Elle se construit en solitude dans une société qui lui semble un vrai désert, déserté d'hommes. Lalla, comme la plupart des personnages des récits de Le Clézio ne cherche pas à se débarrasser de la solitude. Au contraire, elle s'effectue à s'y complait, cette exclusion pour elle est une décision incontournable.

Cette figure féminine de Le Clézio entretient de relations privilégiées avec les éléments de la nature (le désert, la mer, la lumière, les insectes etc.). C'est dans la symbiose avec ces éléments naturels que Lalla atteint une solitude meilleure que celle qu'elle va éprouver dans la ville moderne de Marseille.

A l'inverse, Lalla éprouve la vraie solitude angoissante lorsqu'elle fuit son désert natal et s'oriente à Marseille, pour échapper à un mariage forcé, là où elle rencontre l'autre désert des hommes qui est plus aride que le désert de sable. Dans la grande ville de Marseille, les hommes perdent la capacité de se communiquer avec l'autrui. 
Chacun vit isolé, exclu, à l'écart, en complète isolement. «La solitude est grande ici, comme sur un navire en plein mer» ${ }^{16}$

Moyennant ces termes, Lalla décrit la vie dans la ville de Marseille, il semble que la solitude imprègne cette grande ville. En outre, cette citation affirme que la solitude dans le monde moderne, Marseille le symbole de ce monde, est omniprésente.

Pour Danilo Martucelli, le solitaire constitue le symbole de la modernité. "C'est celui qui, contraint de devenir comme les autres, n'y parvient que rarement, et encore davantage, celui qui, y étant parvenu, ne peut que vivre son refus personnel au milieu d'un profond sentiment de confusion identitaire» ${ }^{17}$

Aspirant à une vie meilleure, Lalla quitte son désert natal et se dirige vers la ville de Marseille. Malheureusement, la ville occidental de Marseille ne représentera pour elle ni la réussite ni l'aspiration à un monde meilleur. Par contre, elle lui représentera la déshumanisation, la faillite, l'inadaptation, la prison. A Marseille Lalla rencontre «les marques de la solitude, de l'abandon» ${ }^{18}$

La solitude n'est pas une sensation éphémère qui hante seulement Lalla. Mais il semble que ce syndrome, voire ce problème de l'isolement est le lot de tout ce qui habite la ville moderne. La solitude est une manifestation qui se propage dans les milieux urbains. Dans Désert, on note bien que tous les personnages à partir du patron de l'hôtel, les clients et même si les passant que Lalla croisait dans les rues(les mendiants, les prostituées, les voyageurs perdus), tous sont seuls, exclus, errants, plutôt isolé.

" [Radicz] connaît tous les mendiants de la ville par leurs noms. Il sait où ils habitent, avec qui ils travaillent, même ceux qui sont plutôt des clochards et qui vivent tous tout seuls» 19

Dans Mondo et autres histoires, on remarque tout d'abord que ces gamins et gamines n'ont, en réalité, aucun lien familiale solide; leurs parents semblent avoir renoncé à exercer leurs rôles, pour diverses raisons. C'est le cas de Mondo, dans la nouvelle éponyme qui porte le titre de son héros, qui erre librement de ville en ville, sans que personne ne sache d'où il venait ni où il va.

«Personne n'aurait pu dire d'où venait Mondo. Il était arrivé un jour, par hasard, dans notre ville, sans qu'on s'en aperçoive, et puis on s'était habitué à lui [...] on ne savait rien de sa famille, ni de sa maison. Peut-être qu'il n'en avait pas» ${ }^{20}$ Mondo illumine la ville par son silence et son innocence pureté puis il disparait un jour sans laisser d'adresse. Sa disparition ainsi que son apparition sont énigmatiques. "Il a mis le feu à ses matelas, dans l'infirmerie, et il a profité de l'affolement pour filer» ${ }^{21}$

Jon, le personnage central de la nouvelle portant le titre La montagne du dieu vivant, a subi la solitude et le vide après avoir quitté la montagne et s'est rentré à la maison. "Jon enfourcha la bicyclette, et il commença à rouler sur le chemin de terre, toujours plus bas. Il ne pensait pas, il ne sentait que le vide, la solitude sans limites. Tandis qu'il pédalait le long du chemin de terre $»^{22}$. Il nous semble que Jon est entré en communion avec le Grand Tout, mais que cette initiation lui a fait mesurer sa solitude.

Daniel, autre figure le clézienne dans l'œuvre sur les enfants Mondo et autres histoires, préfère la vie d'isolement « mais c'était un garçon qui ne parlait pas 
beaucoup. Il ne se mêlait pas aux conversations des autres» ${ }^{23}$. Il vit en marge de la société, refuse les normes de la société moderne, rejette les liens avec les autres. Il aime vit tout seul «il n'avait pas d'amis, il ne connaissait personne et personne ne le connaissait. Peut-être qu'il préférait que ce soit ainsi, pour ne pas être lié» ${ }^{24}$

Il est évident que «htous les enfants de Mondo et autres histoires sont des êtres errants, nomades ou fugueurs ; leurs parents -s'ils en ont- se trouvent relégué dans des rôles très secondaires» ${ }^{25}$. Cette citation, extraite d'une étude consacrée à l'œuvre de Mondo et autres histoires effectuée par Christophe Konaté, nous affirme que la séparation familiale est l'un des points communs qui hantent la plupart des personnages le cléziens ainsi que l'une des causes de la solitude.

A vrai dire, dans le recueil de Mondo et autres histoires, en tant que paratexte, le titre, la gravure des Bains mystérieux qui nous montre trois hommes dont chacun vit tout seul à l'écart dans une grande tour blanche, le reflet du soleil dans l'eau sur la page de la couverture présentent toutes les directions de la lecture. Le lecteur sait dès le début que la solitude constitue le fond et le thème principal de cette œuvre. Mais il est à constater à priori que l'œuvre le clézienne nous présente «la solitude comme une absence d'une relation authentique avec le monde, et non comme une séparation des autres» ${ }^{26}$

Aussi dans son roman La Ronde et autres faits divers, que dans Désert et dans Mondo et autres histoires, le personnage le clézien est un personnage solitaire qui vit à l'écart. C'est le cas de David, le personnage principal dans le roman. «Mais cela lui est égal, il n'a plus peur de la solitude, il ne peut plus craindre le monde, ni les regards de gens, parce qu'il connait maintenant la porte qui conduit vers son frère Edouard, vers sa cachette secrète d'où on ne revient jamais» ${ }^{27}$. David est un enfant solitaire qu'au lieu d'aller à l'école décide d'errer dans les quartiers de la nouvelle ville. Il symbolise la solitude de l'exclus.

Notre réflexion sur l'étude du syndrome de la solitude dans Désert et Mondo et autres histoires de Le Clézio nous mènera à une interrogation des lieux, la mise en écart de différents paysages en tant que l'incubateur de ces personnages solitaires, ainsi que le rapport de ces personnages avec ces lieux de l'écart.

\section{Le lieu de l'écart, l'incubateur du solitaire}

Dans $\underline{\text { Désert }}$ ainsi que dans $\underline{\text { Mondo et autres histoires, }}$ la ville moderne représente le lieu de l'écart le plus dominant. Un lieu où le personnage éprouve la solitude, l'exclusion, le vide, l'éloignement. En outre, «dans son acceptation concrète, l'écart se définit par rapport à un lieu, partie circonscrite du passage. Il fait signe à une mise à part et à un éloignement, une certaine distance d'un lieu à un autre» ${ }^{28}$

Dans son œuvre, La Poétique de l'espace, Gaston Bachelard jette la lumière sur la relation entre l'espace et l'être humain isolé. Il écrit : "Il semble alors que c'est par leur immensité que les deux espaces: l'espace de l'intimité et l'espace du monde deviennent consonants. Quand s'approfondit la grande solitude de l'homme. Les deux immensité se touchent, se confondent» ${ }^{29}$.

Le lieu de l'écart est tout d'abord lié à un mouvement d'éloignement et de fuite comme nous le constatons dans Désert et Mondo et autres histoires. Lalla, dans Désert, fuit son désert natal et choisit de s'éloigner et partir vers la ville de Marseille. Durant ce voyage d'initiation, plutôt d'éloignement, l'auteur nous montre comment la 
ville de Marseille devient un lieu véritable d'écart et d'isolement. Lalla y trouve la solitude et y trouve aussi un autre désert qui est différent de ces ancêtres les hommes bleus.

Comme nous l'avons signalé plus haut, le personnage le clézien, par sa nature et son statut, est un personnage exclu s'évoluant en marge de la société. La demeure d'Es Ser, le personnage impressionnant de Désert tenant à la fois du réel et du rêve, est « un tombeau blanc, simple comme une coquille d'ouf posé sur la terre rouge $»^{30}$. Cette habitation-tombeau, par sa forme de coquille d'œuf, forme un écart dans l'écart du désert.

On remarque bien aussi dans Désert le lieu de l'écart privilégié à partir de la plage où Lalla passe des journées entières toute seule. Cette plage donne naissance à toutes sortes des chemins qui «vont à perte de vue le long des dunes grises, entre les broussailles, ceux qui font une courbe et retournent en arrière, ceux qui font jamais nulle part ${ }^{31}$.

Pour mettre l'accent sur la relation entre la plage, le lieu de l'écart, et Lalla et aussi pour refléter la solitude profonde de cet espace, Le Clézio signale que «elle [Lalla] reconnait ses propres traces dans le sable» ${ }^{32}$.

Tout au long de ses œuvres, Le Clézio consacre une attention particulière aux cachettes, aux collines et aux dunes, c'est-à-dire aux lieux à l'écart.

De même, à Marseille pour s'éloigner de la ville, Lalla dévie dans différentes cachettes et se dirige inconsciemment vers les collines en s'éloignant des rues de la ville pour échapper au carcan de cette ville. Lalla part souvent à la recherche d'espaces vides qui lui permettent de s'isoler, lui permettait ainsi de faire renaître son désert natal. «Lalla monte sur les collines pour tout voir, puis elle redescend $»^{33}$.

Ainsi pour Mondo, les cachettes, outre qu'elles sont des lieux d'écart, représente l'asile auquel il refugie pour échapper à la société urbaine corrompue : «Mondo pensait à toutes ses bonnes cachettes, toutes celles qu'il connaissait, au bord de la mer, dans les rochers blancs, entre les brise-lames, ou bien dans le jardin de la Maison de Lumière et d'Or. [...] c'était comme s'il ne pouvait plus quitter cet endroit, sur le trottoir, contre ce morceau de mur, comme si ses jambes ne pouvaient plus marcher $\rangle^{34}$

L'œuvre le clézienne traduit la volonté de ses personnages de s'écarter de la pression de la société, du carcan de la ville. Tous ses personnages cherchent la solitude pour s'évader de cette hérésie de la société de consommation. Echapper à la solitude dans la société moderne, c'est, en fait, échapper à l'être, dans un mouvement de fuite, de voyage vers un autre monde plus meilleur, c'est-à-dire le monde naturel. C'est pourquoi le personnage le clézien qui souffre l'enfer moderne fuit le monde moderne.

\section{Conclusion}

A vrai dire, la solitude n'est pas un thème dont il faut repérer la trace, mais un problème, voire une problématique épineuse qui inquiète beaucoup d'écrivains contemporains, en tête d'eux notre romancier J. M. G. Le Clézio. Nous espérons traiter et trouver, dans des recherches ultérieurs, la solution de cette problématique qui hante le personnage le clézien, ainsi que nous qui vivent dans cette enfer de la ville. 
La solitude de l'homme au sien de la société urbaine a pour conséquence une sensation d'exclusion, d'isolement, voire d'exil. Celui-ci a contribué, de sa part, à approfondir cette crise d'incommunicabilité entre l'homme et l'enfer moderne de la société.

\section{BIBLIOGRAPHIE:}

1 Cavallero (Claude) et Thibault (Bruno), Les Cahiers J.M.G. Le Clézio numéro 2, Reprotechnique, Paris, P.7.

2 «Une enfance africaine», propos recueillis par Jérôme Garcin, L'événement du jeudi, 21 mars 1991

3 Définition de la solitude : fait social ou effet Clinique, George Jovelet psychiatre des hôpitaux. Journée débat de 10 Mars 2012 sur la solitude, UDAF de l'Aisne, p. 1

4 Le Clézio (J. M. G), Le Déluge, Paris, Gallimard, Coll. L'Imaginaire, 1966, p. 260.

5 Amar (Ruth), Les Structures de la solitude dans l'œeuvre de J.M.G. Le Clézio. Ed. Laballery, Paris, 2004, p. 5.

6 Ibid., p. 109.

7 Jack Messy - Psychanalyste, Co-fondateur et ancien Directeur de ESPACE Gérontologie ESPACES FORMATIONS, 224 avenue du Maine, 75014 Paris, La solitude, Février 1997, p.1.

8 Marie-Chantal Doucet est sociologue et travailleuse sociale. Professeure à l'Université d'Ottawa, elle enseigne les fondements théoriques du travail social et dirige un séminaire sur la sociologie de la santé mentale. Elle possède également une longue expérience clinique dans le champ de la santé mentale. Ses intérêts de recherche portent sur l'individu contemporain ainsi que le développement de la recherche clinique en sciences humaines.

9 Doucet (Marie-Chantal), Solitude et société contemporaine : une sociologie clinique de l'individu et du rapport à l'autre, Canada, Presses de l'université du Québec, 2007, p. 5.

10 Rollo (Fellenberger), Existence- A new dimension en psychiatry and psychology, New York, Basic Books, 1958, p. 56. In La structure de la solitude dans l'ouvre de J.M.G. Le Clézio, OP.CIT. p. 144.

11 Cavallero (Claude), Le Clézio, témoin du monde, Paris, éd. Calliopées, 2009, p.331.

12 Le Clézio (J. M. G.), Le thème de la solitude dans l'œuvre d'Henri Michaux, Diplôme d'études supérieures-Lettres modernes, Aix-en-Provence, 1964.

13 Lohste (Pierre), Conversations, Paris, Mercure de France, pp.58-59. In Les structures de la solitude dans l'œuvre de J.M.G. Le Clézio, p.7

14 Amar (Ruth), Les Structures de la solitude dans l'euvre de J.M.G. Le Clézio, Op.Cit., p.109.

15 Corinne (François), Jean-Marie Gustave Le Clézio: Désert, Paris, Bréal, 2000, p.52.

16 Le Clézio (J. M. G.) Désert. Paris, Gallimard, 1980, p. 287

17 Martucelli (Danilo), Sociologies de la modernité, l'itinéraire du XXe siècle, Paris, Gallimard, coll. « Folio essais », 1999, p. 423.

18 Le Clézio (J. M. G.) Désert. OP.CIT. p. 307

19 Ibid., P. 278 
20 Le Clézio (J.M.G.), Mondo in Mondo et autres histoires, Paris, Gallimard, 1978, P.11.

21 Le Clézio (J.M.G.), Mondo in Mondo et autres histoires, OP.CIT, P. 76.

22 Ibid., p. 146

23 Ibid.,_P. 167.

24 Ibid., P. 168.

25 Konaté(Christophe), Mondo et autres histories de J.M.G. Le Clézio, Paris, Gallimard, 2006, P.325

26 Boulos (Miriam Stendal), Chemins pour une approche poétique du monde, le roman selon J.M.G. Le Clézio, Paris, Gallimard, 1999, p.115.

27 Le Clézio (J. M. G.), La Ronde et autres faits divers, Paris, Gallimard, Coll. Folio Essais, 1990, p. 245.

28 Amar (Ruth), Les Structures de la solitude dans l'œuvre de J.M.G. Le Clézio, Op.Cit. p.9.

29 Bachelard (Gaston), La Poétique de l'espace, Pais, PUF, 1957, p. 184.

30_Le Clézio (J. M. G.) Désert. OP.CIT, p. 205.

31 Ibid, p. 76.

32 Le Clézio (J. M. G.) Désert. OP.CIT, p. 77.

33 Ibid, p. 308.

34 Le Clézio (J.M.G.), Mondo in Mondo et autres histoires, OP.CIT, P. 73. 